\title{
Article \\ Switchable and Dual-Tunable Multilayered Terahertz Absorber Based on Patterned Graphene and Vanadium Dioxide
}

\author{
Hongyao Liu ${ }^{1,2}$, Panpan Wang ${ }^{1}$, Jiali Wu ${ }^{1}$, Xin Yan ${ }^{1, *}{ }^{\mathbb{D}}$, Xueguang Yuan ${ }^{1}{ }^{\mathbb{D}}$, Yangan Zhang ${ }^{1}$ and Xia Zhang ${ }^{1}$ \\ 1 State Key Laboratory of Information Photonics and Optical Communications, Beijing University of Posts and \\ Telecommunications, Beijing 100876, China; liuhongyao1230@gmail.com (H.L.); panpanono@163.com (P.W.); \\ Flowing_Kinga@bupt.edu.cn (J.W.); yuanxg@bupt.edu.cn (X.Y.); zhang@bupt.edu.cn (Y.Z.); \\ xzhang@bupt.edu.cn (X.Z.) \\ 2 School of Information and Communication Engineering, Beijing University of Posts and Telecommunications, \\ Beijing 100876, China \\ * Correspondence: xyan@bupt.edu.cn
}

check for

updates

Citation: Liu, H.; Wang, P.; Wu, J.; Yan, X.; Yuan, X.; Zhang, Y.; Zhang, X. Switchable and Dual-Tunable Multilayered Terahertz Absorber Based on Patterned Graphene and Vanadium Dioxide. Micromachines 2021, 12, 619. https://doi.org/ $10.3390 / \mathrm{mi1} 2060619$

Academic Editor: Shideh Kabiri Ameri

Received: 20 April 2021

Accepted: 25 May 2021

Published: 27 May 2021

Publisher's Note: MDPI stays neutral with regard to jurisdictional claims in published maps and institutional affiliations.

Copyright: (c) 2021 by the authors. Licensee MDPI, Basel, Switzerland. This article is an open access article distributed under the terms and conditions of the Creative Commons Attribution (CC BY) license (https:/ / creativecommons.org/licenses/by/ $4.0 /)$.

\begin{abstract}
In this paper, a switchable and dual-tunable terahertz absorber based on patterned graphene and vanadium dioxide is proposed and analyzed. By controlling the Fermi level of graphene and the temperature of vanadium dioxide, the device's function can be switched and its absorbing properties can be tuned. When the vanadium dioxide is in an insulator state, the device can be switched from near-total reflection (>97\%) to ultra-broadband absorption $(4.5-10.61 \mathrm{THz})$ as the Fermi level of graphene changes from 0 to $0.8 \mathrm{eV}$. When the vanadium dioxide is changed to a metal state, the device can act as a single-band absorber (when the Fermi level of graphene is $0 \mathrm{eV}$ ) and a dual-band absorber with peaks of $4.16 \mathrm{THz}$ and $7.3 \mathrm{THz}$ (when the Fermi level of graphene is $0.8 \mathrm{eV}$ ). Additionally, the absorber is polarization-insensitive and can maintain a stable high-absorption performance within a $55^{\circ}$ incidence angle. The multilayered structure shows great potential for switchable and tunable high-performance terahertz devices.
\end{abstract}

Keywords: multilayered; switchable; dual-tunable; terahertz; patterned graphene; phase-change material vanadium dioxide

\section{Introduction}

The efficient absorption of incident waves in terahertz (THz) bands is of significance for a wide range of applications such as imaging, food quality control, communication, spectroscopy, and sensing devices [1-6]. Metamaterial THz technology has been widely used and investigated in absorbers [7-11]. Graphene is considered one of the most promising metamaterials for tunable $\mathrm{THz}$ absorbers since its conductivity can be easily changed either by manipulating the Fermi energy through chemical doping or by applying a gate voltage [12-15]. Owing to its distinctive electromagnetic and optical properties, graphene is widely considered one of the most effective and promising metamaterials for designing tunable $\mathrm{THz}$ absorbers. Different broadband absorbers made of graphene with patterned disks and ribbons have been widely studied [7,16-19]. Multilayer structure graphene-based absorbers and dual-band absorbers have also been proposed and analyzed [1,20-24]. However, the tunability of graphene absorbers is still limited since, in practice, the Fermi energy level can only be tuned within a small range of $0-0.9 \mathrm{eV}[25,26]$. Moreover, most of the absorbers exhibit a single and unswitchable function that cannot meet various applications.

An efficient approach for realizing switchable and tunable terahertz absorbers is to combine other materials with graphene. This achieves switchable functionality and provides extra tunability. Vanadium dioxide $\left(\mathrm{VO}_{2}\right)$ is an important phase change material that represents an insulator-to-metal transition. Its conductivity can be increased by four to five orders of magnitude under light, thermal approaches, and external stimuli $[27,28]$. When its temperature exceeds the temperature required for phase transition $\left(68^{\circ} \mathrm{C}\right)$, a sharp change 
in conductivity occurs until a conductivity of $2 \times 10^{5} \mathrm{~S} / \mathrm{m}$ is attained. This phase change leads to a reversible transition of infrared light from transmission to reflection $[29,30] . \mathrm{VO}_{2}$ can undergo remarkable changes in its electromagnetic characteristics and can also be applied in tunable broadband $\mathrm{THz}$ absorber designs [31,32]. In this work, we designed a multilayered $\mathrm{THz}$ absorber based on the combination of patterned graphene and $\mathrm{VO}_{2}$. By controlling the Fermi levels of graphene and the temperature of $\mathrm{VO}_{2}$, the device's function can be switched and its absorbing properties can be tuned. When $\mathrm{VO}_{2}$ is in an insulator state, the device can be switched from near-total reflection ( $>97 \%)$ to ultra-broadband absorption $6.11 \mathrm{THz}(4.5-10.61 \mathrm{THz})$ as the Fermi level of graphene changes from 0 to $0.8 \mathrm{eV}$. When $\mathrm{VO}_{2}$ is transmitted to a metallic state, the device can act as a single-band absorber with an absorption band of $0.84 \mathrm{THz}$ (when the Fermi energy level of graphene is $0 \mathrm{eV}$ ) and a dual-band absorber with absorption peaks of $4.16 \mathrm{THz}$ and $7.3 \mathrm{THz}$ (when the Fermi energy level of graphene is $0.8 \mathrm{eV}$ ). Moreover, the absorber is polarization-insensitive and can maintain a stable high-absorption performance within a $60^{\circ}$ incidence angle. In this work, the absorption mechanism is analyzed first with an impedance matching concept [33] and then investigated by electric field distribution. Our results indicate that the device shows great potential for switchable and tunable terahertz applications.

\section{Materials and Model}

\subsection{Patterned Graphene}

As a 2D material, the surface impedance of graphene is characterized by Kubo's formula [34]:

$$
\begin{gathered}
\sigma_{g}\left(f, \mu_{\mathrm{c}}, \tau, T\right)=\frac{j e^{2}\left(2 \pi f-j \tau^{-1}\right) \hbar}{\pi \hbar^{2}}\left[\frac{1}{\left(2 \pi f-j \tau^{-1}\right)^{2}} \int_{0}^{\infty} \varepsilon\left(\frac{\partial f_{d}(\varepsilon)}{\partial \varepsilon}-\frac{\partial f_{d}(-\varepsilon)}{\partial \varepsilon}\right) d \varepsilon\right. \\
-\int_{0}^{\infty} \frac{f_{d}(-\varepsilon)-f_{d}(\varepsilon)}{\left(2 \pi f-j \tau^{-1}\right)^{2}-4(\varepsilon / \hbar)^{2}} d \varepsilon
\end{gathered}
$$

where $f_{d}(\varepsilon)=\left[\exp \left(\varepsilon-\frac{\mu_{\mathrm{c}}}{K_{\mathrm{B}} T}\right)+1\right]^{-1}$ is the Fermi-Dirac distribution, and $\mu_{\mathrm{c}}$ and $\tau$ are the chemical potential and the relaxation time of graphene, respectively. $\hbar, K_{\mathrm{B}}$, and $T$ are the reduced Planck constant, the Boltzmann constant, and the temperature in Kelvin, respectively. In addition, the surface conductivity of graphene can be divided into two parts: $\sigma_{g}=\sigma_{\text {intra }}+\sigma_{\text {inter }}$, where $\sigma_{\text {inter }}$ is the inter-band conductivity and $\sigma_{\text {intra }}$ is the intra-band conductivity. Compared with $\sigma_{\text {intra }}$, the inter-band conductivity $\left(\sigma_{\text {inter }}\right)$ in the terahertz band can be safely neglected based on the Pauli exclusion principle. Finally, the surface conductivity of graphene can be approximated to be a Drude model [35]:

$$
\sigma_{g}\left(f, \mu_{\mathrm{c}}, \tau, T\right) \approx \sigma_{\text {intra }}\left(f, \mu_{\mathrm{c}}, \tau, T\right)=\frac{e^{2} \mu_{\mathrm{c}}}{\pi \hbar^{2}} \frac{j}{\left(2 \pi f+j \tau^{-1}\right)}
$$

The surface conductivity of graphene can change by controlling its chemical potential. The relation of $\mu_{\mathrm{c}}$ and bias voltage $\left(V_{g}\right)$ can be expressed by the following approximate form [36]:

$$
\mu_{\mathrm{c}}=\hbar v_{\mathrm{F}} \sqrt{\frac{\pi \varepsilon_{0} \varepsilon_{h} v_{g}}{e h}}
$$

where $\varepsilon_{h}$ is the permittivity of the dielectric layer, $\varepsilon_{0}$ is the permittivity of the vacuum, $v_{\mathrm{g}}$ is the bias voltage, $e$ is the electron charge, and $v_{\mathrm{F}}$ is the Fermi velocity. The surface impedance $\left(Z_{g}\right)$ of the graphene can be written as follows [37]:

$$
Z_{g}=\frac{1}{\sigma_{g}}
$$

In our simulations, we assumed a relaxation time of $\tau=0.1 \mathrm{ps}$ and a temperature of $T=300 \mathrm{~K}$. The finite element method was used to investigate the absorption performance of the proposed absorber in the frequency range of 1 to $11 \mathrm{THz}$. We numerically calculated 
the real and imaginary parts of the surface impedance of the graphene film with different chemical energies. The results are shown in Figure 1. The real part remains constant with an increase in the frequency, whereas the imaginary part increases monotonously as the frequency increases. It can also be observed that the real and imaginary parts of the surface impedance decrease as the chemical potential of graphene increases. Tuning can be achieved by controlling the gate voltage using an ion-gel top configuration [38]. This changes the surface impedance of the patterned graphene. Therefore, a tunable absorber is achievable.

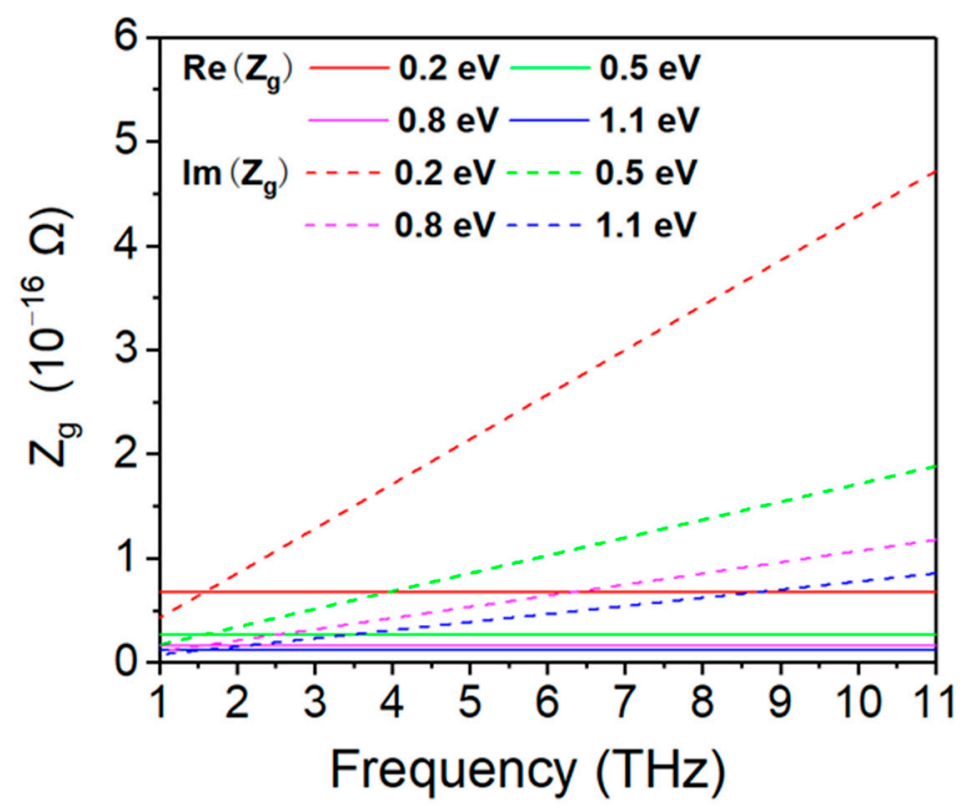

Figure 1. Surface impedance versus frequency of patterned graphene.

\subsection{Phase-Change Material $\mathrm{VO}_{2}$}

Within $\mathrm{THz}$ range, the relative permittivity of $\mathrm{VO}_{2}$ can be described by the Drude model [39]:

$$
\begin{gathered}
\varepsilon_{\mathrm{VO}_{2}}(\omega)=\varepsilon_{\infty}-\frac{\omega_{\mathrm{P}}^{2}\left(\sigma_{\mathrm{VO}_{2}}\right)}{\omega^{2}+i \gamma \omega} \\
\omega_{\mathrm{P}}^{2}\left(\sigma_{\mathrm{VO}_{2}}\right)=\frac{\sigma_{\mathrm{VO}_{2}}}{\sigma_{0}} \omega_{\mathrm{P}}^{2}\left(\sigma_{0}\right)
\end{gathered}
$$

where $\varepsilon_{\infty}$ is the infinite frequency permittivity with a numerical value of $12, \gamma=5.75 \times 10^{13} \mathrm{~s}^{-1}$ expresses the damping frequency, and $\omega$ is the angular frequency of the incident wave. Additionally, $\sigma_{0}=3 \times 10^{5} \mathrm{~S} / \mathrm{m}$ and $\omega_{p}\left(\sigma_{0}\right)=1.4 \times 10^{15} \mathrm{rad} / \mathrm{s}$. The conductivity of $\mathrm{VO}_{2}$ spans several orders of magnitude when it is changed from an insulator state to a metallic state by thermal and electrical stimuli [40]. $\mathrm{As}_{\mathrm{VO}_{2}}$ is difficult to control during its transition, two stable states of $\mathrm{VO}_{2}$ were taken into consideration for investigating the absorber. For the conductivity of $\mathrm{VO}_{2}\left(\sigma_{\mathrm{VO}_{2}}\right)$ in Equation (6), we considered values of $10 \mathrm{~S} / \mathrm{m}$ and $2 \times 10^{5} \mathrm{~S} / \mathrm{m}$ for the insulator state and metallic state, respectively [30,41]. A sharp increase in conductivity makes the impedance of an absorber mismatched with the free space impedance of some frequencies, and the absorption can be changed by this transition.

\subsection{Model Design}

Figure 2a shows a schematic view of the proposed structure for this paper. The dualtunable $\mathrm{THz}$ absorber is composed of two graphene film layers and one $\mathrm{VO}_{2}$-gold based layer, sandwiched between a $\mathrm{SiO}_{2}$ layer with a relative permittivity of 2.25 [42]. Each layer can be fabricated by stacking a chemical vapor deposition (CVD)-grown patternedgraphene on the dielectric spacer $\left(\mathrm{SiO}_{2}\right)$ which is supported by a back plate. The thicknesses of the $\mathrm{SiO}_{2}$ layers are $h_{1}, h_{2}$, and $h_{3}$ from bottom to top, respectively. Here, the dielectric 
layers act as resonance absorption spaces. The bottom of the structure is a gold mirror with a thickness of $d_{5}=0.2 \mu \mathrm{m}$. This gold mirror is thick enough to block the propagation of electromagnetic waves and reflect the energy reduced in graphene. Absorption is strengthened by this part of the gold mirror. The first layer is an ultra-thin patterned graphene film, formed by subtracting four rectangular films from a disk with a radius of $2.7 \mu \mathrm{m}$. The width of the interval between these different parts is $d_{1}$. For the second layer, eight rectangular films with a side length of $l_{1}$ and interval width $d_{2}$ were subtracted from a square patterned graphene. The third layer consists of a crossed-shaped $\mathrm{VO}_{2}$ with four gold parts in the corners of the square. The square has a thickness of $d_{4}$, and the side length of the outsider square is $l_{1}$ and the side length of the hollow part is $l_{4}$. The absorption of the different layers affects each other, which enhances overall absorption and expands bandwidth. The electric field of the patterned graphene design is concentrated to the edge because of the local surface plasmon resonance that contributes to the absorption. Narrow intervals added to the designed pattern can increase the edge of the graphene and can enhance its absorption. Moreover, the whole structure is symmetrical to ensure that the absorber is insensitive to polarizations and incident angles within a large range. The parameters of the absorber proposed are introduced below. Figure $2 \mathrm{~b}$ displays an ion-gel gate device for tuning the chemical level of the graphene [43]. Additionally, an important factor in the $\mathrm{VO}_{2}$ 's transition is temperature. It can be tuned using electrifying and terahertz radiation. Adulteration is sometimes used to change the transition temperature in order to suit practical working conditions.

(a)

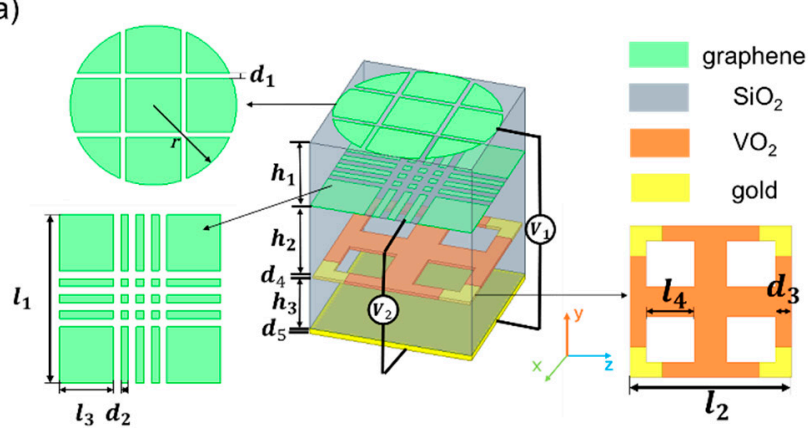

(b)

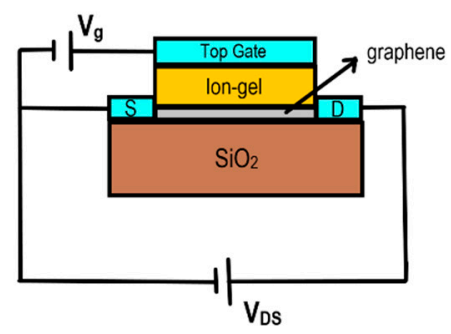

Figure 2. (a) A schematic view of the graphene- $\mathrm{VO}_{2}$ based absorber and a top view of each layer. (b) Instructure of the ion-gel gate device that controls the chemical potential of the graphene. Here, $\mathrm{S}=$ source and $\mathrm{D}=$ drain.

The initial chemical potential of the graphene is assumed as $\mu_{\mathrm{c}}=0.8 \mathrm{eV} . \mathrm{VO}_{2}$ is in an insulator state with a conductivity of $10 \mathrm{~S} / \mathrm{m}$. Both the finite element method and ANSYS HFSS are applied to analyze the absorption characteristics of the absorber. A THz wave is applied perpendicular to the upper graphene surface. The periodic boundary conditions are used for the $x$-direction and the y-direction, and the Floquet ports are set in the z-direction. The absorption of the absorber can be calculated as follows:

$$
A(f)=1-R(f)-T(f)
$$

where $R(f)=\left|S_{11}(f)\right|^{2}$ is the reflection and $T(f)=\left|S_{21}(f)\right|^{2}$ is the transmission. The $S$ parameters are obtained from ANSYS HFSS. Due to the ground gold mirror, the $T(f)$ is zero and the expression of the absorption can be simplified as follows:

$$
A(f)=1-R(f)
$$

Therefore, a relatively high absorption can be obtained when the reflection efficiency is close to zero. Furthermore, the equivalent input impedance $\left(Z_{\text {in }}\right)$ should match the free space impedance $\left(Z_{0}\right)$. The size of the patterned graphene and $\mathrm{VO}_{2}$-gold layers and the thickness of the dielectric layers can affect the input impedance of the whole 
structure. To facilitate simulation work, the selection and optimization of the proposed structure parameters were conducted by using the Optimetrics function in ANSYS HFSS. The parameters proposed in Figure 2a are listed in Table 1 for an intuitive view. These parameters make the impedance of the structure match the free space within the high absorption range.

Table 1. Parameters of the proposed structure $(\mu \mathrm{m})$.

\begin{tabular}{cccccc}
\hline Parameter & Size & Parameters & Size & Parameter & Size \\
\hline$d_{1}$ & 0.18 & $d_{5}$ & 0.2 & $l_{1}$ & 1.05 \\
$d_{2}$ & 0.26 & $l_{1}$ & 5.4 & $h_{1}$ & 2.15 \\
$d_{3}$ & 0.5 & $l_{2}$ & 5.25 & $h_{2}$ & 2 \\
$d_{4}$ & 0.5 & $l_{3}$ & 1.8 & $h_{3}$ & 2.5 \\
\hline
\end{tabular}

\section{Results and Discussion}

In this section, both the broadband absorption of the proposed structure and the absorptions of different parts of the structure are displayed and analyzed in Figure $3 a, b$, respectively. The TE incident wave is chosen. Under the simulation conditions set above, the whole structure shows an absorption of over $90 \%$ in the frequency range of $4.5-10.61 \mathrm{THz}$ (6.11 THz bandwidth), which is wider than most of the existing broadband $\mathrm{THz}$ absorbers proposed. Additionally, the chemical energy of the two graphene layers is set to $0.8 \mathrm{eV}$, thereby making it more convenient to realize in the experiment. The central frequency $\left(f_{\mathrm{c}}\right)$ is $7.56 \mathrm{THz}$, and the fractional bandwidth is $80.87 \%$. It can be concluded that the real part of the impedance is close to 1 and that the imaginary part is close to 0 , which demonstrates that the impedance of the absorber matches the free space. To further discuss the function of multilayered structures in achieving broadband absorption, the absorption curves of both single-layer and multi-layer structures are displayed in Figure 3b. Two graphene layers with different patterns appear to have two distinctive ranges of absorption over $90 \%$. With only one layer of patterned graphene, an absorption of over $90 \%$ is relatively narrow. When two layers of patterned graphene act together, the bandwidth has an obvious broadening. The simulated results indicate that a multilayer structure can broaden the absorption range of the absorber.

(a)

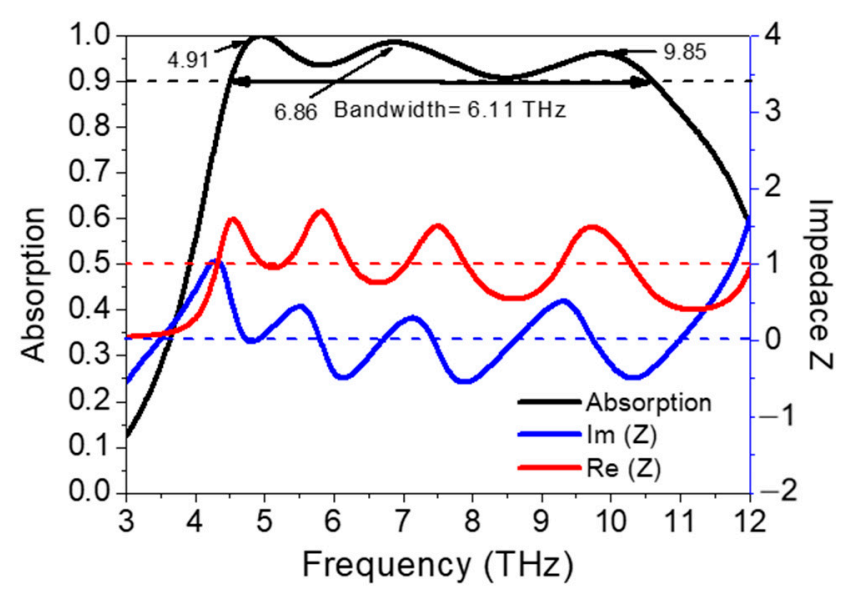

(b)

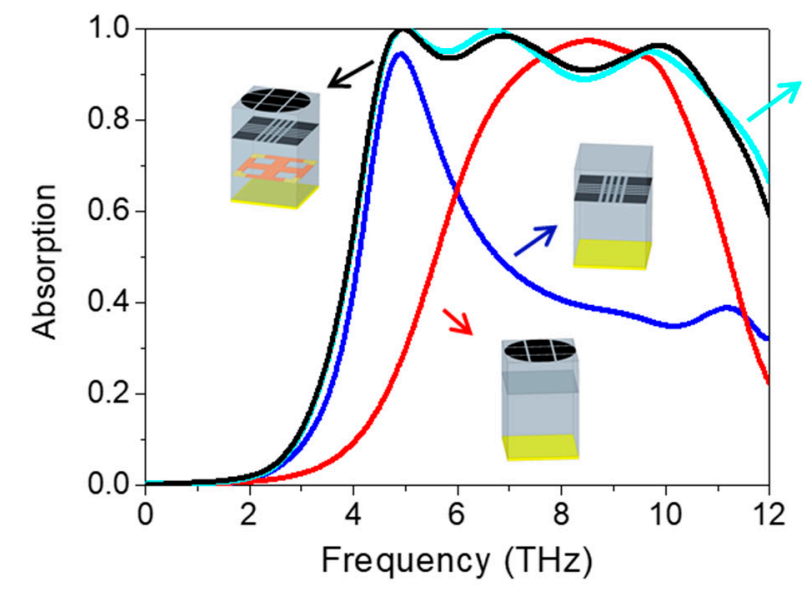

Figure 3. (a) The absorption curves and normalized impedances of the absorber when $\mu_{\mathrm{c}}=0.8 \mathrm{eV}$ and $\mathrm{VO}_{2}$ is in an insulator state. (b) The absorption curves of different parts of the absorber: the black line represents the absorber with three layers; the blue-green line represents the absorber with two top layers; the blue line represents the absorber with the second layer; and the red line represents the absorber with the top layer. 
In order to further disclose the mechanism of the absorber, electric field distributions of the absorber have been plotted. The frequencies corresponding to the absorption peaks of the curves plotted in Figure 3a are listed in Table 2 to make browsing the information more intuitive.

Table 2. Absorption peaks in broadband absorption.

\begin{tabular}{|c|c|c|c|c|c|c|}
\hline$\mu_{\mathrm{c}}$ and $\mathrm{VO}_{2}$ State & \multicolumn{6}{|c|}{ Absorption Peaks and Corresponding Frequencies } \\
\hline \multirow{2}{*}{$0.8 \mathrm{eV}$ Insulator } & \multicolumn{2}{|c|}{ Peak I } & \multicolumn{2}{|c|}{ Peak II } & \multicolumn{2}{|c|}{ Peak III } \\
\hline & $4.91 \mathrm{THz}$ & $100 \%$ & $6.86 \mathrm{THz}$ & $98.50 \%$ & $9.85 \mathrm{THz}$ & $96.35 \%$ \\
\hline
\end{tabular}

In order to further discuss the mechanism of the broadband absorption mode of the absorber, the electric field distributions from both an $x-y$ perspective and $y-z$ perspective are given in Figure 4. In the left of Figure 4, the electric field distribution is observed from an x-y perspective with different frequencies of $4.91 \mathrm{THz}, 6.86 \mathrm{THz}$, and $9.85 \mathrm{THz}$, respectively. The upper half represents the distribution of the top circle layer, while the lower half represents the electric field distribution in the second graphene layer. In general, we found that the electric field primarily concentrates near the edges of graphene with all resonant frequencies, which indicates that local electric resonance occurs in these areas. Electric dipole resonance is also activated at the edges of the graphene layer [44]. As shown in the top graphene layer, when the simulation resonant frequency increases, edges with the peak value electric field intensity are decreased while other parts of graphene tend to have an increase in the electric field intensity. The electric field intensity becomes more dispersed and covers the entire graphene, which can be explained as a result of the local surface plasmon resonance of graphene being excited by the incident $\mathrm{THz}$ wave [45]. In the second graphene layer, the electric field distribution has a trend of decreasing first and then increasing with increasing simulated frequency. Similar to the results in the top layer, the peak value of the electric field at the edge of graphene decreases and an increase occurs in other parts of the graphene layer. The incident wave excites carriers and induces tangential electric fields on both the top graphene layer and the second graphene layer.
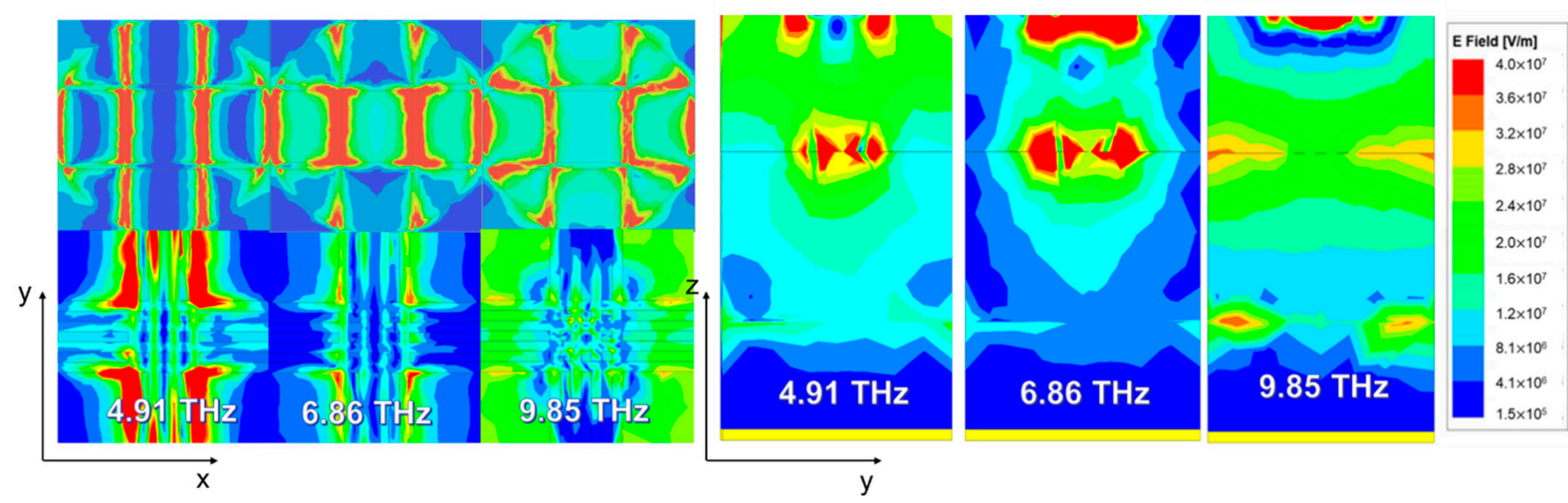

Figure 4. The $\mathrm{x}-\mathrm{y}$ perspective and $\mathrm{y}-\mathrm{z}$ perspective of electric field distribution when $\mu_{\mathrm{c}}=0.8 \mathrm{eV}$ and $\mathrm{VO}_{2}$ is in an insulator state at absorption peaks of $4.91 \mathrm{THz}, 6.86 \mathrm{THz}$, and $9.85 \mathrm{THz}$.

The electric field intensity distributions in the y-z plane of the absorber at each peak frequency are plotted in the right side of Figure 4. By comparing the electric field intensity of different parts, the important part for incident wave absorption at each resonant frequency 
can be distinguished. In principle, the energy consumption and optical loss in materials can be represented by the following equation:

$$
A(\lambda)=2 \pi \frac{c}{\lambda} \varepsilon^{\prime \prime} \int_{V}\left|E_{l}\right|^{2} d V
$$

where $\varepsilon^{\prime \prime}$ is the imaginary part of the dielectric permittivity, $V$ is the volume of the material, $V$ is the electric field inside the material, and $c$ is the speed of light in a vacuum. The electric field is trapped in graphene layers, in the $\mathrm{VO}_{2}$ layer, and in the $\mathrm{SiO}_{2}$ dielectric layers. However, the absorption effect of the dielectric layers is less effective than other parts, corresponding to electric distribution. This can be explained by Equation (9); the imaginary parts of graphene and $\mathrm{VO}_{2}$ permittivity are larger than the dielectric layers within the $\mathrm{THz}$ range. In resonant frequencies of $4.91 \mathrm{THz}$ and $6.86 \mathrm{THz}$, energy consumption mainly occurs in the top and second graphene layers. As the resonant frequency rises to $9.85 \mathrm{THz}$, the energy consumption of the $\mathrm{VO}_{2}$ layer is strengthened since the electric field intensity increased.

The switchable function of the $\mathrm{THz}$ absorber is achieved by tuning both the Fermi energy level and the phase transition property of the $\mathrm{VO}_{2}$. The structure acts as a broadband $\mathrm{THz}$ absorber when $\mu_{\mathrm{c}}$ is $0.8 \mathrm{eV}$ and $\mathrm{VO}_{2}$ is in an insulator state and as a dual-band absorber when $\mu_{\mathrm{c}}$ is $0.8 \mathrm{eV}$ and $\mathrm{VO}_{2}$ is in a metallic state. Additionally, when the voltage applied to the graphene layers is set to 0 , the device acts as a single-band absorber when the $\mathrm{VO}_{2}$ is in a metallic state and as a broadband reflector when the $\mathrm{VO}_{2}$ is in an insulator state. The reflectivity is greater than $97 \%$ because the transmission coefficient of the absorber is 0 and the absorption is less than 0.03. As shown in Figure 5a, the dual-band absorption has absorption peaks of $4.16 \mathrm{THz}$ and $7.3 \mathrm{THz}$ and the single-band absorption has an absorption band of 4.81-5.65 $(0.84 \mathrm{THz})$. The simulated results show that the switchable working characteristics of the structure can be controlled and changed significantly.

(a)

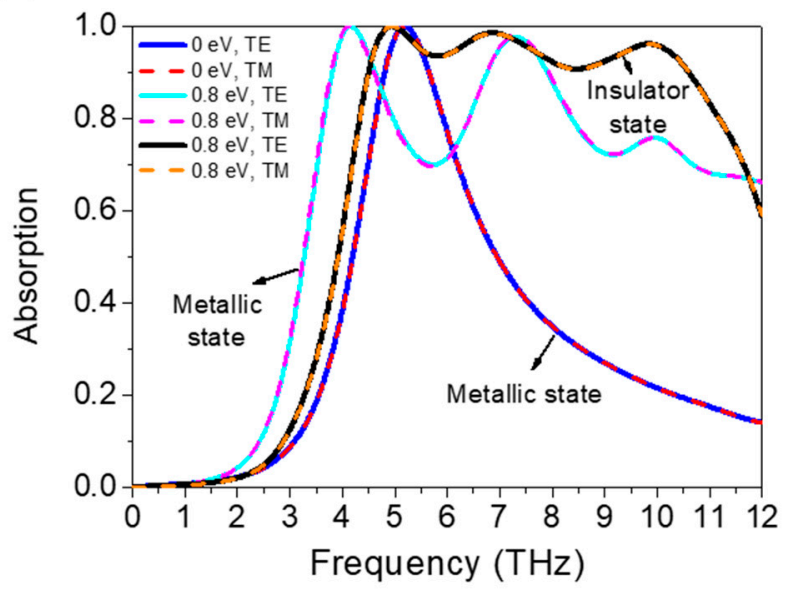

(b)

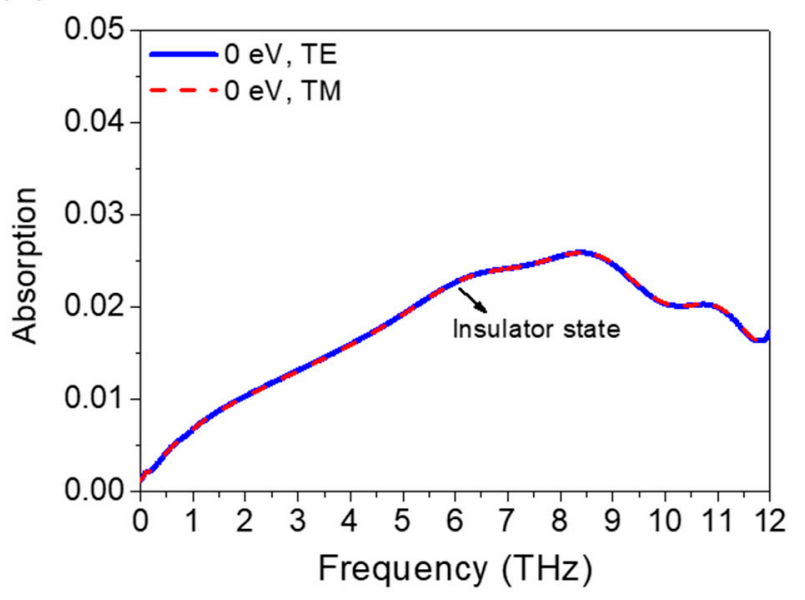

Figure 5. (a) Broadband absorption of an absorber with $0.8 \mathrm{eV}$ Fermi energy levels and insulator $\mathrm{VO}_{2}$ states, dual-band absorption with $0.8 \mathrm{eV}$ Fermi energy and metallic $\mathrm{VO}_{2}$ state, and single-band absorption with $0 \mathrm{eV}$ and metallic absorption (TE and TM polarization). (b) Absorption with the Fermi energy level $0 \mathrm{eV}$ and $\mathrm{VO}_{2}$ insulator state (TE and TM polarization).

It is of great importance to design absorbers equipped with polarization insensitivity and tolerance within a wide incident angle $(\theta)$ range. In this section, absorptions of the proposed absorber are plotted in contour maps to analyze their insensitivities in both the incident angles and polarization angles. In Figure 6, three switchable absorption modes of the absorber (broadband absorber, dual-band absorber, and single- and narrow-band absorber) are discussed with respect to different polarization angles $\varphi$ and incident angles $\theta$. In Figure $6 \mathrm{a}, \mathrm{d}, \mathrm{g}$, the absorption spectra of the broadband, dual-band, and single narrow 
band are shown with different polarization $\varphi$. From our results, it can be concluded that the absorber is polarization-insensitive. This can be ascribed to the asymmetrical design of the structure. For broadband absorption, spectra with TE and TM polarized waves are represented in Figure $6 \mathrm{~b}, \mathrm{c}$, respectively. Three absorption peaks in this absorption mode can keep the absorption over $80 \%$ up to a $55^{\circ}$ incident angle for the TE mode and up to a $60^{\circ}$ incident angle for the TM mode. As shown in Figure 6e,f, for the TE polarized incident wave, the two absorption peaks of the dual-band absorber can keep absorption over $80 \%$ up to an incident angle of $60^{\circ}$. The dual-band absorber can maintain stable absorption up to $65^{\circ}$ for the TM mode, and the absorption peaks tend to have a blue shift with an increasing incident angle. In Figure $6 \mathrm{~h}, \mathrm{i}$, the absorption spectra of relatively narrow and single-band absorbers with TE and TM modes are displayed, respectively. Both of these absorbers can keep the absorption peak over $80 \%$ up to an incident angle of $65^{\circ}$. Great tolerance for incident angle is obtained by the subwavelength structure, which can facilitate the match between the structure and the wave from free space. Overall, the switchable $\mathrm{THz}$ absorber proposed in this work possesses an incident angle insensitivity and a polarization insensitivity in different absorption modes.

(a)

(d)

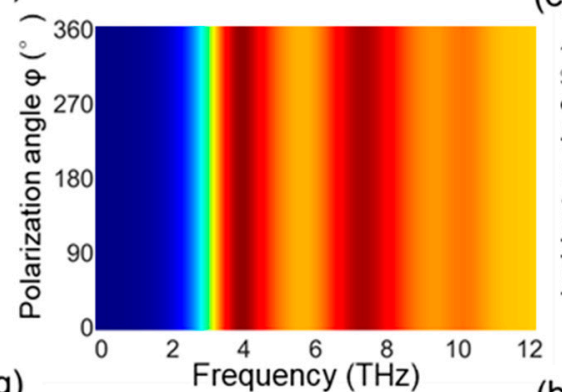

(g)

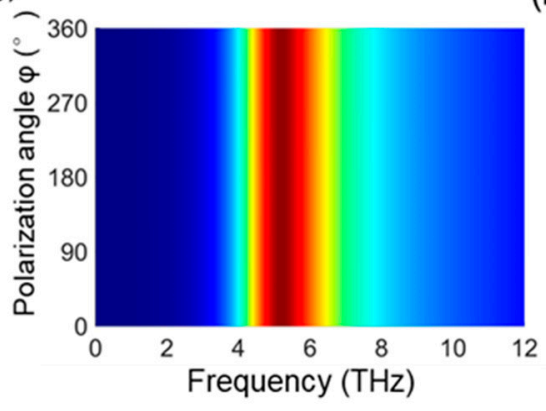

(b)

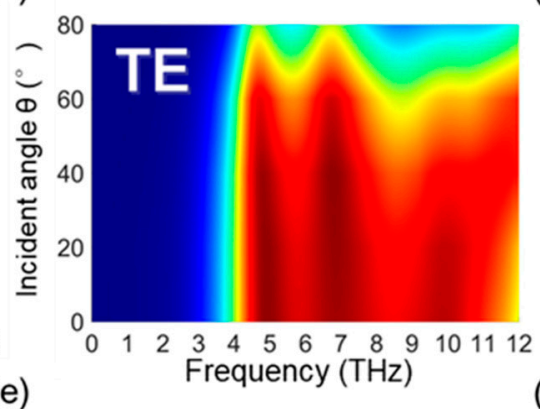

e)
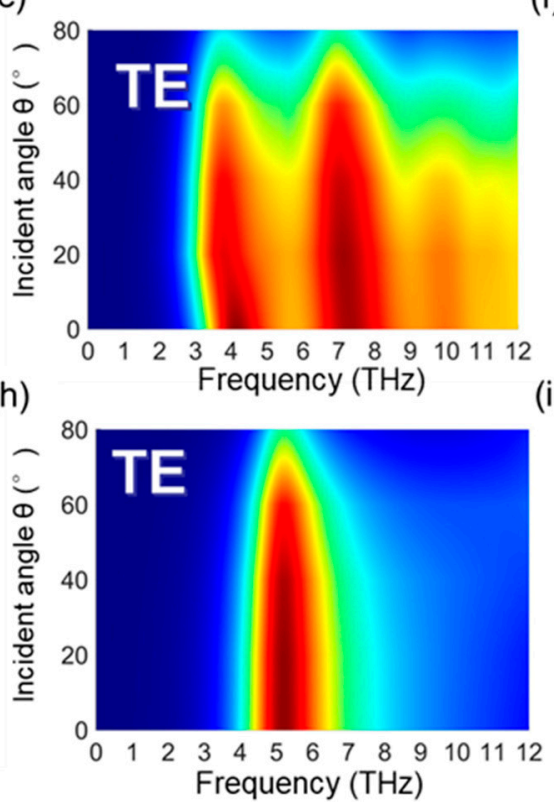

(c)

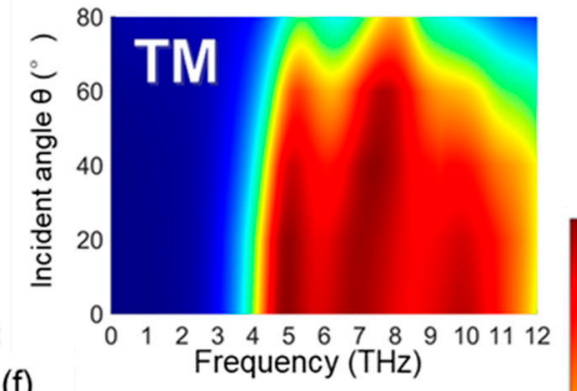

(f)
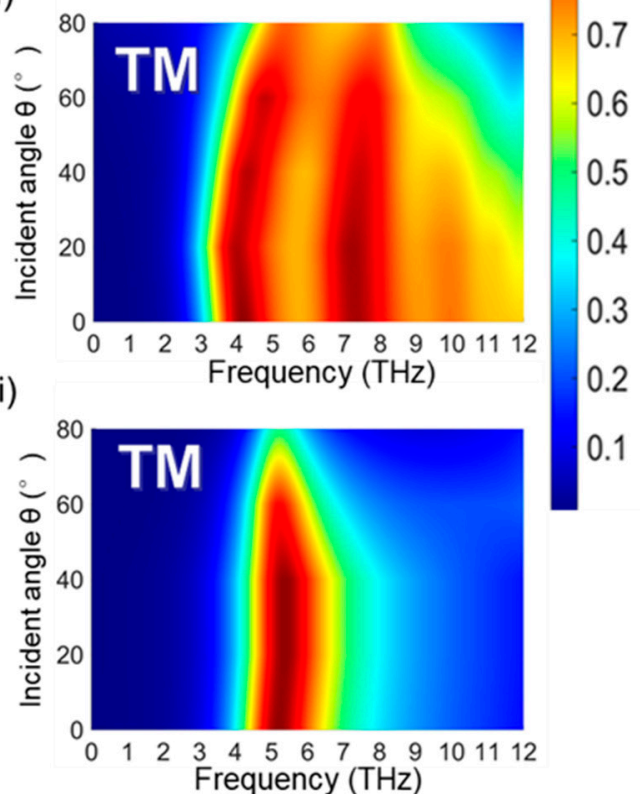

Figure 6. Absorption spectra with different polarization angles $(\varphi)$ and incident angles $(\theta)$ and frequency of the incident wave from 0 to $12 \mathrm{THz}$ : (a-c) broadband absorption when $\mu_{\mathrm{c}}=0.8 \mathrm{eV}$ and $\mathrm{VO}_{2}$ is in an insulator state; (d-f) dual-band absorption when $\mu_{\mathrm{c}}=0.8 \mathrm{eV}$ and $\mathrm{VO}_{2}$ is in a metallic state; (g-i) single-band and relatively narrow absorption when $\mu_{\mathrm{c}}=0 \mathrm{eV}$ and $\mathrm{VO}_{2}$ is in a metallic state.

Finally, we discuss the tunable function for the broadband absorber of Fermi energy level of the graphene and the relaxation time $\tau$. In practical situations, different energy levels $\left(\mu_{\mathrm{c}}\right)$ for two graphene layers are hard to implement. Therefore, the Fermi energy 
was set the same for each graphene layer. In Figure $7 \mathrm{a}$, the values of $\mu_{\mathrm{c}}$ are set to $0.2 \mathrm{eV}$, $0.5 \mathrm{eV}$, and $0.8 \mathrm{eV}$, respectively. Because the relaxation time is in direct proportion to the chemical potential $\mu_{\mathrm{c}}$, the following expression is given: $\tau=\frac{\mu \mu_{\mathrm{c}}}{e v_{\mathrm{F}}^{2}}$. Here, $\mu$ is the electron mobility decided by the quality of the graphene and is fixed on $1250 \mathrm{~cm}^{2} / V \mathrm{~s}$. Therefore, the relaxation time in Figure 7a is 0.025 ps, 0.0625 ps, and $0.1 \mathrm{ps}$ for $0.2 \mathrm{eV}, 0.5 \mathrm{eV}$, and $0.8 \mathrm{eV}$, respectively. Here, the $\mathrm{VO}_{2}$ is in an insulator state. The absorption curves of both $\mathrm{TE}$ and TM polarized waves are then calculated. The results indicate that the structure proposed can support different polarized incident waves. As $\mu_{\mathrm{c}}$ increases, the absorption increases gradually and absorption peaks start to appear. Additionally, the quantity of the absorption peaks increases and the broadband absorption appears. The relationship between the Fermi energy $\mu_{\mathrm{c}}$ and the conductivity of graphene is introduced in Section 2.1. The higher the value of $\mu_{\mathrm{c}}$, the greater the surface impedance of the graphene. This change leads to an increase in absorption. Moreover, the relationship between central frequency $\left(f_{c}\right)$ and consecutive Fermi energy levels varies within a range of $0.75 \mathrm{eV}-0.85 \mathrm{eV}$. Tuning $\mu_{\mathrm{c}}$ in the range of $0.8 \mathrm{eV} \pm 0.05 \mathrm{eV}$, the $f_{\mathrm{c}}$ of the broadband absorber can be tuned linearly. It can be concluded that the graphene also plays an important role in controlling the structure. The relaxation time $(\tau)$ of the patterned graphene is investigated because it can be changed by the quality of the graphene [46].

(a)

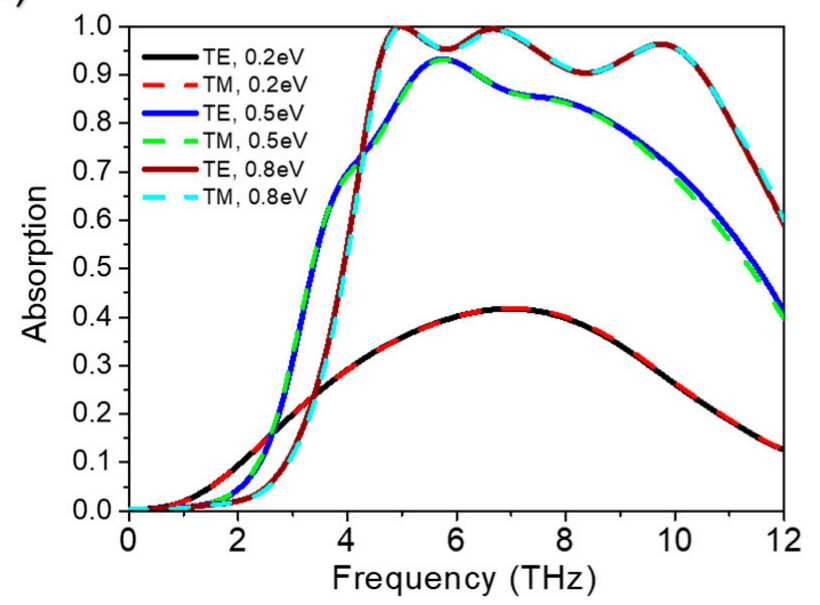

(b)

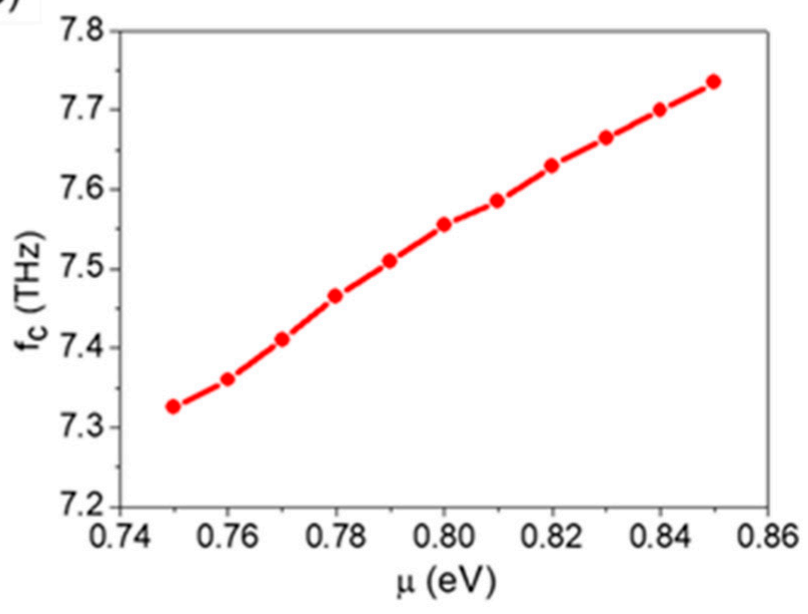

Figure 7. (a) Absorptions with different polarizations; Fermi energy levels $\left(\mu_{\mathrm{c}}\right)$ of $0.2 \mathrm{eV}, 0.5 \mathrm{eV}$, and $0.8 \mathrm{eV}$; and $\tau=\frac{\mu \mu_{c}}{e v_{\mathrm{F}}^{2}}$.

(b) Central frequencies with consecutive Fermi energy levels from $0.75 \mathrm{eV}$ to $0.85 \mathrm{eV}$.

In order to compare our absorber with other absorbers in the references, we listed the main properties of broadband absorbers in Table 3. Compared with other structures, the hybrid absorber exhibits both a significantly larger bandwidth and a higher fractional bandwidth with a small number of layers, showing great potential for miniaturized broadband THz applications.

Table 3. Comparison of important properties of $\mathrm{THz}$ absorbers.

\begin{tabular}{|c|c|c|c|c|c|}
\hline References & $\begin{array}{c}\text { Absorption } \\
\text { Bandwidth (THz) }{ }^{1}\end{array}$ & Material & Layers & $\begin{array}{c}\text { Stable Incident } \\
\text { Angle }\end{array}$ & $\begin{array}{l}\text { Polarization } \\
\text { Insensitivity }\end{array}$ \\
\hline [19] & $2.76(2.14-4.90)$ & Graphene & 1 & Up to $60^{\circ}$ & Insensitive \\
\hline [11] & $3.7(1.95-5.65)$ & Gold & 1 & Up to $20^{\circ}$ & Insensitive \\
\hline [1] & $0.76(4.80-5.56)$ & Graphene & 3 & $\mathrm{Up}$ to $60^{\circ}$ & Insensitive \\
\hline [18] & $2.2(0.6-2.8)$ & Graphene & 3 & Up to $60^{\circ}$ & Insensitive \\
\hline [22] & $3.5(6.98-9.1)$ & Graphene & 3 & Up to $60^{\circ}$ & Insensitive \\
\hline
\end{tabular}


Table 3. Cont.

\begin{tabular}{|c|c|c|c|c|c|}
\hline References & $\begin{array}{c}\text { Absorption } \\
\text { Bandwidth (THz) }{ }^{1}\end{array}$ & Material & Layers & $\begin{array}{c}\text { Stable Incident } \\
\text { Angle }\end{array}$ & $\begin{array}{l}\text { Polarization } \\
\text { Insensitivity }\end{array}$ \\
\hline \multirow{3}{*}{ This work } & $6.11(4.5-10.61)$ & $\begin{array}{c}\text { Graphene and } \mathrm{VO}_{2} \\
\text { (0.8 eV insulator state) }\end{array}$ & 3 & $\begin{array}{l}\text { TE up to } 55^{\circ} \\
\text { TM up to } 60^{\circ}\end{array}$ & Insensitive \\
\hline & $\begin{array}{l}0.83(3.78-4.61) \\
1.19(6.75-7.94)\end{array}$ & $\begin{array}{l}\text { Graphene and } \mathrm{VO}_{2} \\
(0.8 \mathrm{eV} \text { metallic state })\end{array}$ & 3 & $\begin{array}{l}\text { TE up to } 60^{\circ} \\
\text { TM up to } 65^{\circ}\end{array}$ & Insensitive \\
\hline & $0.84(4.81-5.65)$ & $\begin{array}{l}\text { Graphene and } \mathrm{VO}_{2}(0 \\
\mathrm{eV} \text { metallic state })\end{array}$ & 3 & $\begin{array}{l}\text { TE up to } 65^{\circ} \\
\text { TM up to } 65^{\circ}\end{array}$ & Insensitive \\
\hline
\end{tabular}

${ }^{1}$ Bandwidth of absorption over $90 \%$.

\section{Conclusions}

In summary, a switchable, dual-tunable $\mathrm{THz}$ absorber based on patterned graphene and phase-change $\mathrm{VO}_{2}$ was proposed and investigated. When $\mathrm{VO}_{2}$ was in an insulator state, the device could be switched from near-total reflection $(>97 \%)$ to high absorption over a wide frequency range $(4.5-10.61 \mathrm{THz})$ as the Fermi level of graphene changed from 0 to $0.8 \mathrm{eV}$. When $\mathrm{VO}_{2}$ was transmitted to a metal state, the device acted as a single-band absorber with absorption band $0.84 \mathrm{THz}$ (when the Fermi energy level of graphene is $0 \mathrm{eV}$ ). When $\mathrm{VO}_{2}$ was in a metal state and the Fermi energy level of graphene was tuned to $0.8 \mathrm{eV}$, the device acted as a dual-band absorber with bands of $3.78-4.61 \mathrm{THz}$ and 6.75-7.94 THz. The absorber was polarization-insensitive and could maintain a stable high absorption performance within a $55^{\circ}$ incidence angle. Moreover, the broadband structure could maintain a stable absorption within a $\tau$ change of $\pm 50 \%$. With respect to the large absorption bandwidth, flexible tunability, and switchable functionality, the device is promising for various terahertz applications.

Author Contributions: Conceptualization, H.L. and P.W.; methodology, H.L., P.W. and J.W.; software, H.L., J.W. and X.Y. (Xueguang Yuan); writing — original draft preparation, H.L. and P.W.; writingreview and editing, H.L. and X.Y. (Xin Yan); supervision, X.Y. (Xin Yan), Y.Z. and X.Z.; project administration, X.Y. (Xin Yan) and X.Z. All authors have read and agreed to the published version of the manuscript.

Funding: This research was funded by the Research Innovation Fund for College Students of Beijing University of Posts and Telecommunications, the Fund of State Key Laboratory of Information Photonics and Optical Communications (Beijing University of Posts and Telecommunications), China (IPOC2020ZZ01 and IPOC2019ZT07), and the National Natural Science Foundation of China (61774021).

Conflicts of Interest: The authors declare no conflict of interest.

\section{References}

1. Cai, Y.J.; Xu, K.D. Tunable broadband terahertz absorber based on multilayer graphene-sandwiched plasmonic structure. Opt. Express 2018, 26, 31693-31705. [CrossRef]

2. Federici, J.; Moeller, L. Review of terahertz and sub terahertz wireless communications. J. Appl. Phys. 2010, 107, 6. [CrossRef]

3. Watts, C.M.; Liu, X.; Padilla, W.J. Metamaterial electromagnetic wave absorbers. Adv. Mater. 2012, 24, OP98-OP120. [CrossRef]

4. Guo, C.; Zhang, J.; Liu, K.; Yuan, X.; Qin, S.; Zhu, Z. Graphene-based perfect absorption structures in the visible to terahertz band and their optoelectronics applications. Nanomaterials 2018, 8, 1033. [CrossRef] [PubMed]

5. Jiang, S.; Li, J.Y.; Li, J.Z.; Zhang, G.Z.; Lin, H.; Yi, F. Genetic optimization of plasmonic metamaterial absorber towards dual-band infrared imaging polarimetry. Opt. Express 2020, 28, 22617-22629. [CrossRef] [PubMed]

6. Abbas, A.; Ali, M.; Ali, F. Ultra high-sensitivity and tunable dual-band perfect absorber as a plasmonic sensor. Opt. Laser Technol. 2020, 127, 106201.

7. Zhang, Y.; Feng, Y.; Zhu, B.; Zhao, J.; Jiang, T. Graphene based tunable metamaterial absorber and polarization modulation in terahertz frequency. Opt. Express 2014, 22, 22743-22752. [CrossRef] [PubMed]

8. Chen, H.T. Interference theory of metamaterial perfect absorbers. Opt. Express 2012, 20, 7165-7172. [CrossRef]

9. Cui, Y.; He, Y.; Jin, Y.; Ding, F.; Yang, L.; Ye, Y.; Zhong, S.; Lin, Y.; He, S. Plasmonic and metamaterial structures as electromagnetic absorbers. Laser Photonics Rev. 2014, 8, 495-520. [CrossRef] 
10. Zhu, J.; Ma, Z.; Sun, W.; Ding, F.; He, Q.; Zhou, L.; Ma, Y. Ultra-broadband terahertz metamaterial absorber. Appl. Phys. Lett. 2014, 105, 021102. [CrossRef]

11. Meng, W.; Lv, J.; Zhang, L.; Que, L.; Zhou, Y.; Jiang, Y. An ultra-broadband and polarization-independent metamaterial absorber with bandwidth of $3.7 \mathrm{THz}$. Opt. Commun. 2018, 431, 255-260. [CrossRef]

12. Geim, A.K.; Novoselov, S.K. The rise of graphene. Nat. Mater. 2007, 6, 183-191. [CrossRef]

13. Zhou, Q.; Liu, P.; Bian, L.; Liu, H.; Liu, C.; Chen, G. Controlling enhanced absorption in graphene metamaterial. Opt. Commun. 2018, 413, 310-316. [CrossRef]

14. Yan, J.; Zhang, Y.; Kim, P.; Pinczuk, A. Electric field effect tuning of electron-phonon coupling in graphene. Phys. Rev. Lett. 2007, 98, 166802. [CrossRef] [PubMed]

15. Bonaccorso, F.; Sun, Z.; Hasan, T.; Ferrari, A.C. Graphene photonics and optoelectronics. Nat. Photonics 2010, 4, 611-622. [CrossRef]

16. Ye, L.; Zeng, F.; Zhang, Y.; Xu, X.; Yang, X.; Liu, Q.H. Frequency-reconfigurable wide-angle terahertz absorbers using single- and double-layer decussate graphene ribbon arrays. Nanomaterials 2018, 8, 834. [CrossRef] [PubMed]

17. Chen, P. and Alu., A. Terahertz metamaterial devices based on graphene nanostructures. IEEE Trans. THz Sci. Technol. 2013, 3 , 748-756. [CrossRef]

18. Biabanifard, S.; Biabanifard, M.; Asgari, S.; Asadi, S.; Yagub, M.C.E. Tunable ultra-wideband terahertz absorber based on graphene disks and ribbons. Opt. Commun. 2018, 427, 418-425. [CrossRef]

19. Du, X.; Yan, F.; Wang, W.; Tan, S.; Zhang, L.; Bai, Z.; Zhou, H.; Hou, Y. A polarization- and angle-insensitive broadband tunable metamaterial absorber using patterned graphene resonators in the terahertz band. Opt. Laser Technol. 2020, 132, 106513. [CrossRef]

20. Huang, M.L.; Cheng, Y.Z.; Cheng, Z.Z.; Chen, H.R.; Mao, X.S.; Gong, R.Z. Based on graphene tunable dual-band terahertz metamaterial absorber with wide-angle. Opt. Commun. 2018, 415, 194-201. [CrossRef]

21. Wang, B.X.; Wang, G.Z.; Wang, L.L. Design of a novel dual-band terahertz metamaterial absorber. Plasmonics 2016, 11, 523-530. [CrossRef]

22. Pan, F.; Fei, L.; Guang, J.; Fei, S.; Dong, L.; Jian, Q. A broadband metamaterial absorber based on multi-layer graphene in the terahertz region. Opt. Commun. 2018, 417, 62-66.

23. Qi, L.; Liu, C.; Ali, S.; Syed, M. A broad dual-band switchable graphene-based terahertz metamaterial absorber. Carbon 2019, 153, 179-188. [CrossRef]

24. Xu, Z.; Wu, D.; Liu, Y.; Liu, C.; Yu, Z.; Yu, L.; Ye, H. Design of a Tunable Ultra-Broadband Terahertz Absorber Based on Multiple Layers of Graphene Ribbons. Nanoscale Res. Lett. 2018, 13, 1-8. [CrossRef]

25. Zhang, Q.; Li, X.; Hossain, M.M.; Xue, Y.; Zhang, J.; Song, J.; Liu, J.; Turner, M.D.; Fan, S.; Bao, Q.; et al. Graphene surface plasmons at the near-infrared optical regime. Sci. Rep. 2015, 4, 6559. [CrossRef]

26. Xu, W.; Zhu, Z.H.; Liu, K.; Zhang, J.F.; Yuan, X.D.; Lu, Q.S.; Qin, S.Q. Toward integrated electrically controllable directional coupling based on dielectric loaded graphene plasmonic waveguide. Opt. Lett. 2015, 40, 1603-1606. [CrossRef]

27. Jepsen, P.U.; Fischer, B.M.; Thoman, A.; Helm, H.; Suh, J.Y.; Lopez, R.; Haglund, R.F.J. Metal-insulator phase transition in a VO2 thin film observed with terahertz spectroscopy. Phys. Rev. B 2006, 74, 205103. [CrossRef]

28. Wen, Q.-Y.; Zhang, H.-W.; Yang, Q.-H.; Xie, Y.-S.; Chen, K.; Liu, Y.-L. Terahertz metamaterials with VO2 cut-wires for thermal tunability. Appl. Phys. Lett. 2010, 97, 021111. [CrossRef]

29. Zang, X.; Cheng, S.; Lin, C.; Zhu, Y.; Zhuang, S. Ultra-broadband terahertz absorption by exciting the orthogonal diffraction in dumbbell-shaped gratings. Sci. Rep. 2015, 5, 8901. [CrossRef] [PubMed]

30. Wang, T.; Zhang, Y.; Zhang, H.; Cao, M. Dual-controlled switchable broadband terahertz absorber based on a graphene-vanadium dioxide metamaterial. Opt. Mater. Express 2020, 10, 369-386. [CrossRef]

31. Song, Z.; Wang, K.; Li, J.; Liu, Q. Broadband tunable terahertz absorber based on vanadium dioxide metamaterials. Opt. Express 2018, 26, 7148-7154. [CrossRef] [PubMed]

32. Liu, H.; Wang, Z.H.; Li, L.; Fan, Y.X.; Tao, Z. Vanadium dioxide-assisted broadband tunable terahertz metamaterial absorber. Sci. Rep. 2019, 9, 5751. [CrossRef] [PubMed]

33. Khavasi, A. Design of ultra-broadband graphene absorber using circuit theory. J. Opt. Soc. Am. B 2015, 32, 1941-1946. [CrossRef]

34. Hanson, G.W. Dyadic Green's functions and guided surface waves for a surface conductivity model of graphene. J. Appl. Phys. 2008, 103, 064302. [CrossRef]

35. Xiao, S.; Wang, T.; Liu, T.; Yan, X.; Li, Z.; Xu, C. Active modulation of electromagnetically induced transparency analogue in terahertz hybrid metal-graphene metamaterials. Carbon 2018, 126, 271-278. [CrossRef]

36. Zhang, Y.; Feng, Y.; Jiang, T.; Cao, J.; Zhao, J.; Zhu, B. Tunable broadband polarization rotator in terahertz frequency based on graphene metamaterial. Carbon 2018, 133, 170-175. [CrossRef]

37. Mishra, R.; Panwar, R.; Singh, D. Equivalent Circuit Model for the Design of Frequency-Selective, Terahertz-Band, Graphene-Based Metamaterial Absorbers. IEEE Mag. Lett. 2018, 9, 1-5. [CrossRef]

38. Hu, H.; Zhai, F.; Hu, D.; Li, Z.; Bai, B.; Yang, X.; Dai, Q. Broadly tunable graphene plasmons using anion-gel top gate with low control voltage. Nanoscale 2015, 7, 19493-19500. [CrossRef]

39. Song, Z.; Deng, Y.; Zhou, Y.; Liu, Z. Terahertz toroidal metamaterial with tunable properties. Opt. Express 2019, $27,5792-5797$. [CrossRef] 
40. Zhang, C.; Zhou, G.; Wu, J.; Tang, Y.; Wen, Q.; Li, S.; Han, J.; Jin, B.; Chen, J.; Wu, P. Active Control of Terahertz Waves Using Vanadium-Dioxide-Embedded Metamaterials. Phys. Rev. Appl. 2019, 11, 054016. [CrossRef]

41. Liu, M.; Xu, Q.; Chen, X.; Plum, E.; Li, H.; Zhang, X.; Zhang, C.; Zou, C.; Han, J.; Zhang, W. Temperature-Controlled Asymmetric Transmission of Electromagnetic Waves. Sci. Rep. 2019, 9, 4097. [CrossRef] [PubMed]

42. Lim, W.X.; Singh, R. Universal behaviour of high-Q Fano resonances in metamaterials: Terahertz to near-infrared regime. Nano Converg. 2018, 5, 1-7. [CrossRef] [PubMed]

43. Chen, C.-F.; Park, C.-H.; Boudouris, B.W.; Horng, J.; Geng, B.; Girit, C.; Zettl, A.; Crommie, M.F.; Segalman, R.; Louie, S.G.; et al. Controlling inelastic light scattering quantum pathways in graphene. Nat. Cell Biol. 2011, 471, 617-620. [CrossRef] [PubMed]

44. Guo, H.; Liu, N.; Fu, L.; Meyrath, T.P.; Zentgraf, T.; Schweizer, H.; Giessen, H. Resonance hybridization in double split-ring resonator metamaterials. Opt. Express 2007, 15, 12095-12101. [CrossRef] [PubMed]

45. Vakil, A.; Engheta, N. Transformation Optics Using Graphene. Science 2011, 332, 1291-1294. [CrossRef] [PubMed]

46. Arsanjani, A.; Biabanifard, M.; Abrishamian, M.S. A novel analytical method for designing a multi-band, polarization-insensitive and wide angle graphene-based THz absorber. Superlattices Microstruct. 2019, 128, 157-169. [CrossRef] 